\title{
Non-muscle myosin heavy chain IIA and IIB interact and co-localize in living cells: Relevance for $\mathrm{MYH}$-related disease
}

\author{
MONICA MARINI ${ }^{1,6}$, MAURIZIO BRUSCHI $^{2}$, ALESSANDRO PECCI $^{3}$, ROBERTA ROMAGNOLI $^{4}$, \\ LUCA MUSANTE $^{2}$, GIOVANNI CANDIANO ${ }^{2}$, GIAN MARCO GHIGGERI ${ }^{2}$, CARLO BALDUINI $^{3}$, \\ MARCO SERI $^{5}$ and ROBERTO RAVAZZOLO ${ }^{1,6}$
}

\begin{abstract}
Laboratories of ${ }^{1}$ Molecular Genetics, and ${ }^{2}$ Nephrology, G. Gaslini Institute, Genova; ${ }^{3}$ Department of Internal Medicine, University of Pavia and IRCCS Policlinico S. Matteo, Pavia; ${ }^{4}$ Department of Physiology, University of Siena, Siena;

${ }^{5}$ Laboratory of Medical Genetics, Department of Internal Medicine, Cardioangiology and Hepatology, University of Bologna, Bologna; ${ }^{6}$ Department of Pediatrics and CEBR, University of Genova, Genova, Italy
\end{abstract}

Received November 18, 2005; Accepted January 9, 2006

\begin{abstract}
Myosins of class II constitute part of a superfamily of several classes of proteins expressed in almost all eukaryotic cell types. Differences in the heavy chains produce three isoforms of class II non-muscle myosins (A, B and C), which are widely distributed in most tissues and thought to be components of the cell motor systems, although specific functional roles are largely unknown. In particular, it is still a matter of debate whether they interact and have overlapping or distinct functions. This argument is relevant not only to cell physiology, but also to human pathology since mutations of the MYH9 gene encoding non-muscle myosin heavy chain II A (NMMHC-A) cause $M Y H 9$-related disease (MYH9-RD), an autosomal dominant disorder characterized by platelet macrocytosis, thrombocytopenia and leukocyte inclusions, variably associated with sensorineural hearing loss, cataracts and/or glomerulonephritis. In this study, we report the results of yeast two-hybrid screening showing that the C-terminals of NMMHC-A and -B interact. This interaction was confirmed by immunoprecipitation in transfected COS-7 cells and in skin fibroblasts naturally expressing both isoforms. Moreover, our immunomorphological study revealed that isoforms A and B co-localize in fibroblasts, erythroblasts and kidney cells. These results suggest that isoforms A and B are strictly related molecules and support the hypothesis that their interrelationship could be involved both in the variability of clinical phenotype and selectivity of tissue damage of MYH9-RD.
\end{abstract}

Correspondence to: Professor Roberto Ravazzolo, Laboratory of Molecular Genetics, G. Gaslini Institute, Largo G. Gaslini 5, 16147 Genova, Italy

E-mail: rravazzo@unige.it

Key words: non-muscle myosins, yeast two hybrid screening, MYH9-related disease

\section{Introduction}

Myosins of class II constitute part of a large superfamily of several distinct classes of proteins expressed in almost all eukaryotic cell types. They include conventional, sarcomeric, smooth muscle, and non-muscle myosins. Class II myosins are composed of two heavy chains and two pairs of light chains, with each of the heavy chains binding an essential and a regulatory light chain. In heavy chains, the N-termini form two globular heads with actin and ATP domains required for motor activity, while the $\alpha$-helical $\mathrm{C}$-termini form a single rod-like tail, which allows the molecules to polymerize into bipolar multimeric filaments (1). For class II non-muscle myosin heavy chains (NMMHCs), the three genes MYH9, MYH1O and MYH14 have been identified and located on chromosomes 22q12.3, 17p13.3 and 19q13.3, respectively, which encode three protein isoforms, NMMHC-A, NMMHC-B, and NMMHC-C. The heavy chains share the same genomic structure, suggesting that they originated from the same ancestral gene.

Despite their wide distribution in most tissues, remarkable differences in isoform expression exist among different cell types. Interesting examples are platelets and mouse $\mathrm{T}$ cells that express only NMMHC-A, while the monkey kidney fibroblast cell line (COS-7), cardiac myocytes from newborn mice and primary cultures of embryonic chicken cardiac myocytes express only NMMHC-B (2-4).

Myosins of class II are essential components of the cell motor system that, in eukaryotes, is involved in several important cell functions such as phagocytosis, maintenance of cell shape and polarity, and intracellular organelle/particle trafficking $(5,6)$. Functional studies on the two best known isoforms, $\mathrm{A}$ and $\mathrm{B}$, reported differences in their biological properties, thus suggesting that they might play different biological roles (7-9). Null mice for myosin IIA or IIB supported this hypothesis in that the former failed to develop a normal patterned embryo (10), while the latter had defects in heart and brain development (2). A recent study, besides confirming that knockout mice are unable to develop, has described hearing loss as the only pathological 
manifestation in $1 / 3$ of mice heterozygous for the targeted disruption of Myh9 (11).

Alterations in class II myosins are relevant to human pathology in that heterozygous mutations of MYH9 or MYH14 have been detected in two families with non-syndromic hearing loss $(12,13)$. Moreover, heterozygous mutations of MYH9 determine a complex disorder named MYH9-related disease (MYH9-RD) (14). At birth, patients present platelet macrocytosis, thrombocytopenia and leukocyte inclusions containing NMMHC-A. Moreover, later in life, some of them develop additional features of sensorineural hearing loss, cataracts and/or glomerulonephritis that sometimes lead to end-stage renal failure. Based on these phenotypic differences, patients were in the past classified as being affected by MayHegglin anomaly, Sebastian syndrome, Fechtner syndrome or Epstein syndrome. However, the absence of correlation between the $22 \mathrm{MYH} 9$ mutations identified so far and clinical pictures, together with the wide phenotypic variability within the same family, prompted the conclusion that these disorders are not distinct entities, but rather a single illness with a continuous clinical spectrum ranging from macrothrombocytopenia to a severe form complicated by deafness, cataracts and renal failure (14). In accordance with the clinical picture, NMMHC-A is expressed in platelets, leukocytes, kidney, and cochlea $(12,15,16)$. Most of the MYH9 mutations identified so far are missense and affect the motor or the coiled-coil domain of NMMHC-A, whereas a few non-sense and frameshift alterations are located in the last exon.

One major, unresolved problem in MYH9-RD is how to justify the remarkable symptomatic variability. To explain this phenomenon, it has been suggested that the clinical picture derives from the joint effect of $M Y H 9$ mutations and additional factors, such as polymorphic variants of proteins interacting with NMMHC-A, but no experimental data have so far supported this hypothesis. Another unsolved question is why MYH9 mutations result in abnormal phenotypes in only a few of the cell types/tissues expressing NMMHC-A.

To elucidate further information on NMMHC-A physiology that could be useful in gaining a better understanding of the pathogenic mechanisms of MYH9-RD, we aimed to identify proteins that interact with NMMHC-A. Here, we describe different genetic, biochemical and immunomorphological studies that demonstrated NMMHC-B to be a strong interactor with NMMHC-A, and discuss the possible implications of this disclosure.

\section{Materials and methods}

Reagents. Sodium dodecyl sulfate (SDS) and sodium deoxycholate were obtained from Fluka Chemie. Protein A/G agarose, protease and phosphatase inhibitors were from Sigma. Acrylamide, N,N'-methylenebisacrylamide, ammonium persulfate, $\mathrm{N}, \mathrm{N}, \mathrm{N}^{\prime} \mathrm{N}$--tetramethylethylenediamine (TEMED), B-mercaptoethanol and molecular weight standards were from Bio-Rad Labs. Non-fat dry milk was obtained from Santa Cruz Biotechnology.

Yeast two-hybrid screening. The MAV203 yeast strain was genetically modified to contain three GAL4 inducible reporter genes, HIS3, URA3 and LacZ, stably integrated in the genome, and used with the ProQuest Two Hybrid System (Invitrogen) following the manufacturer's instructions. The bait construct was prepared by cloning a 1,200-bp fragment of $M Y H 9$, including exons 34 to 40 in the pDBLeu vector that corresponded to amino acids 1645-1960 of the NMMHC-A C-terminal end of the coiled-coil domain, after PCR amplification with oligonucleotide primers designed to contain restrictions sites, SalI at the 5' end and NotI at the $3^{\prime}$ end, in the appropriate positions to allow in-frame fusion with the DNA binding domain of the GALA transcription factor. A commercial ProQuest HeLa cell library (Invitrogen), in which cDNAs were cloned in the pPC86 vector fused in frame with the activation domain of the GAL4 transcription factor, was utilized. The screening was performed by sequential transformation of MAV203 yeast cells with the pDBLeuMYH9 construct and library pPC86 plasmids. Colony selection, plasmid DNA isolation from positive clones, and specificity tests were performed according to the ProQuest Invitrogen instruction manual. Positive clones were sequenced and screened by Blast analysis at the National Center for Biotechnology Information database.

Direct interaction assay was carried out by testing different fragments from the $M Y H 9$ gene inserted in the pDBLeu vector: a) the fragment corresponding to $1645-1960$ amino acids of the coiled-coil domain of NMMHC-A, as above; b) a fragment corresponding to 1298-1960 amino acids of the coiled-coil domain; and c) a fragment corresponding to 1-724 amino acids of the head domain. These were co-transformed in yeast cells with the MYH1O fragment corresponding to 1168 1644 amino acids of the coiled-coil domain of NMMHC-B. Positive interaction was detected by the reactivity of the three reporter genes, His, Ura and LacZ.

Antibodies. Isoform-specific antibodies were used for the immunodetection of NMMHCs. For NMMHC-A, we used rabbit polyclonal PR-B440P (Covance Research Products) raised against a synthetic peptide corresponding to a unique sequence of the C-terminus of human NMMHC-A $(17,18)$, and rabbit polyclonal BT-561 (Biomedical Technologies) raised against purified human platelet myosin. For NMMHC-B, we used the mouse monoclonal antibody (MoAb) CMII23 (Developmental Studies Hybridoma Bank, University of Iowa) raised against chick brain myosin that selectively recognizes an amino acid sequence specific to the C-terminus of human NMMHC-B (19). The following primary antibodies were also used: mouse MoAb AC-74 against $\beta$-actin (Sigma), mouse monoclonal antibody anti-Flag (Sigma), and rabbit polyclonal anti-myc (Santa Cruz).

Cells, cell lysates and cell transfection. COS-7 (monkey kidney fibroblast-like cells) were obtained from the American Type Culture collection (ATCC). Primary cell cultures of human kidney tubular epithelial cells (HKT) and human fibroblasts were developed as previously described (20). GECs, primary cultures of glomerular podocytes, were established and immortalized as previously described $(21,22)$. Immortalized GEC cells were cultured in DMEM containing $25 \mathrm{mM}$ glucose, $10 \%$ inactivated FCS, $100 \mathrm{U} / \mathrm{ml}$ penicillin, and $100 \mu \mathrm{g} / \mathrm{ml}$ streptomycin at $37^{\circ} \mathrm{C}$ and then used for immunofluorescence experiments. 
For immunoprecipitation, cells at confluence were washed in ice-cold phosphate-buffered solution (PBS) and lysed in ice-cold modified RIPA buffer $(10 \mathrm{mM}$ Tris- $\mathrm{HCl} \mathrm{pH} 7.8$, $100 \mathrm{mM} \mathrm{NaCl}, 0.5 \%$ NP-40, $0.5 \%$ sodium-deoxycholate and $0.1 \%$ SDS) with $1 \mathrm{mM}$ EDTA plus phosphatase and protease inhibitors. After $30 \mathrm{~min}$ at $4^{\circ} \mathrm{C}$, the mixture was centrifuged at $15,000 \mathrm{x} \mathrm{g}$ for $15 \mathrm{~min}$, and the supernatant was aliquoted and immediately frozen in liquid nitrogen prior to storage at $-80^{\circ} \mathrm{C}$.

Platelets from healthy donors were centrifuged at $600 \mathrm{x} \mathrm{g}$ for $15 \mathrm{~min}$ at room temperature and then washed 5 times with PBS (2 mM EGTA) at room temperature. Finally, the pellet was solubilized in 10\% SDS, 3\% DTE, $40 \mathrm{mM}$ Tris and $0.1 \mathrm{mM}$ EDTA, and boiled for $5 \mathrm{~min}$. This mixture was aliquoted and immediately frozen in liquid nitrogen prior to storage at $-80^{\circ} \mathrm{C}$.

For transfection experiments, $M Y H 9$ encoding cDNA was cloned in the pCMVTag-2B expression vector (Stratagene) containing the Flag epitope tag by PCR using oligonucleotide primers designed to contain the restrictions sites, SalI at the $5^{\prime}$ end and NotI at the $3^{\prime}$ end. MYH1O encoding cDNA was cloned in the pCDNA $3.1 /$ myc-His vector (Invitrogen) with the myc and polyhistidine epitopes by PCR using oligonucleotide primers designed to contain the restriction sites, Sal1 at the 5' end and NotI at the 3 'end.

COS-7 cells were transiently transfected or co-transfected with $\mathrm{MYH9}$ and $\mathrm{MYH1O}$ expression vectors using PolyFect reagent (Qiagen) according to the manufacturer's instructions. The cells were seeded at $1.6 \times 10^{6}$ per $100-\mathrm{mm}$ diameter dish and allowed to attach overnight and then co-transfected using a total of $5 \mu \mathrm{g}$ plasmid DNA for each plate. Cells were harvested $48 \mathrm{~h}$ after transfection, washed and lysed in buffer containing $150 \mathrm{mM} \mathrm{NaCl}, 50 \mathrm{mM}$ Tris- $\mathrm{HCl} \mathrm{pH}$ 8.0, $1 \%$ NP40 and protease inhibitor cocktail (Roche).

Co-immunoprecipitation studies. Cell extracts were subjected to pre-clearing for $1 \mathrm{~h}$ by incubation with $50 \mu \mathrm{l}$ of a $50 \%$ slurry of protein A agarose (Amersham Pharmacia Biotech) at $4^{\circ} \mathrm{C}$ with gentle rocking. After centrifugation, an aliquot of supernatant $(500 \mu \mathrm{g} / \mathrm{ml})$ was incubated overnight at $4^{\circ} \mathrm{C}$ with $10 \mu \mathrm{g} / \mathrm{ml}$ of the appropiate antibody with gentle rocking. For the capture of immunocomplexes, $50 \mu 1$ of protein A agarose was added and incubated overnight at $4^{\circ} \mathrm{C}$ with gentle rocking. The supernatant was discarded, and the pellet was resuspended in Laemmli reducing sample buffer (23) and boiled for $5 \mathrm{~min}$ to dissociate the immunocomplexes from agarose beads. Beads were precipitated by centrifugation, and the supernatant was subjected to SDS-PAGE. The antibodies used were CMII23 against NMMHC-B, rabbit polyclonal PR-B440P against NMMHC-A, rabbit polyclonal anti-myc, and mouse monoclonal antibody M2 anti-Flag (Sigma). Precipitates were washed 3 times with PBS containing $0.1 \%$ NP40 and resuspended in SDS-reduced sample buffer.

Protein electrophoresis and immunoblotting. An aliquot of lysate supernatant was de-lipidated and cleaned with a solution consisting of tri-n-butylphosphate:acetone:methanol $(1: 12: 1)$ and then cooled in ice (24). The precipitate was pelleted by centrifugation at $2,800 \mathrm{x}$ g for $20 \mathrm{~min}$ at $4^{\circ} \mathrm{C}$, washed again with the same de-lipidizing solution, centrifuged and then air-dried. Finally, the pellet was dissolved in Laemmli reducing sample buffer (23).

After separation in SDS-PAGE gels with 6-16T\% gradient, the proteins were transferred by semidry Western blotting onto nitrocellulose membranes to verify protein identity. The membranes were blocked with $5 \%$ non-fat dry milk in Trisbuffered saline (TBS) overnight at $4^{\circ} \mathrm{C}$. After incubation overnight at $4^{\circ} \mathrm{C}$ with moAb CMII23 diluted 1:5000, AC-74 anti- $\beta$-actin diluted 1:5000, PR-B440P diluted 1:5000, polyclonal anti-myc diluted 1:2500 or moAb M2 anti-flag diluted 1:2500, membranes were washed in TBS with $0.15 \%$ Tween-20 and incubated with horseradish peroxidase conjugated anti-mouse IgG diluted 1:50,000 or anti-rabbit IgG diluted 1:30,000 for $2 \mathrm{~h}$ at room temperature. Detection was performed using the chemiluminescent SuperSignal West Pico kit from Pierce.

Immunofluorescence studies and confocal microscopy. Immunofluorescence labeling was performed on preparations of human cultured cells (podocyte cell lines, HKT cells and fibroblasts described above) grown on a glass coverslip, and on human bone marrow touch preparations. Bone marrow smears from 5 different subjects were evaluated. In all cases, marrow aspiration had been performed as part of the diagnostic procedure and gave normal results. Each subject gave informed consent for the study. Specimens were double-labeled for NMMHC-A and -B using PR-B440P or BTI-561 in combination with the CMII23 antibody. PR-B440P and BTI561 gave identical results. The following antibody dilutions were used: 1:400 for both PR-B440P and BTI-561, and 1:30 for CMII23. Briefly, cells were fixed with $4 \%$ paraformaldehyde (Sigma) for $10 \mathrm{~min}$, rinsed with TBS, and permeabilized with $0.1 \%$ Triton-X-100 (Bio-Rad) in TBS 1\% BSA for 5 min. After a thorough washing with TBS, slides were incubated with the primary antibody and then with Alexa Fluor 488conjugated goat anti-mouse or Alexa Fluor 594-conjugated goat anti-rabbit antibody (Molecular Probes), diluted 1:200 $(10 \mu \mathrm{g} / \mathrm{ml})$. The entire sequence was repeated for the second labeling. Specimens were then incubated with a solution of Hoechst 33258 penthahydrate (Molecular Probes) for nuclear counterstaining, rinsed with buffer and mounted in Mowiol 4-88 (Calbiochem). Negative controls were performed by replacing the primary antibody with an isotype-matched mouse moAb or an irrelevant rabbit polyclonal antibody.

Confocal microscopy analysis was performed through the TCS SPII confocal laser scanning microscopy system (Leica), equipped with a Leica DM IRBE inverted microscope. The following laser line emissions were used: argon/visible $488 \mathrm{~nm}$ for Alexa Fluor 488, helium/neon $543 \mathrm{~nm}$ for Alexa Fluor 594, and argon/UV $364 \mathrm{~nm}$ for Hoechst. The band amplitude to detect fluorescence was between 500 and $550 \mathrm{~nm}$ for Alexa Fluor 488, 590 and $670 \mathrm{~nm}$ for Alexa Fluor 594, and 400 and $500 \mathrm{~nm}$ for Hoechst. Confocal optical sections were performed every $400 \mathrm{~nm}$.

Immunohistochemistry. Immunohistochemistry on renal bioptic samples was performed by double-labeling for NMMHC-A and $\mathrm{B}$, using enhanced polymer one-step staining reagents for immune-enzyme double-staining as described (25). Accordingly, 4- to 5- $\mu \mathrm{m}$ tissue sections were de-waxed, 
Interaction of NMMHC-IIA with NMMHC-IIB (1168-1644 aa)

A

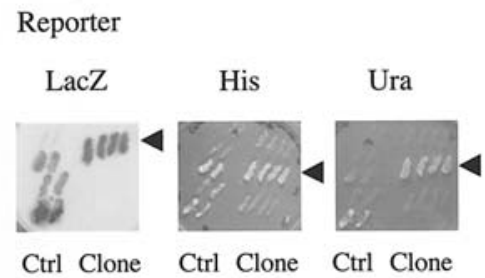

B

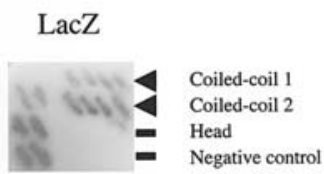

Ctrl Clone
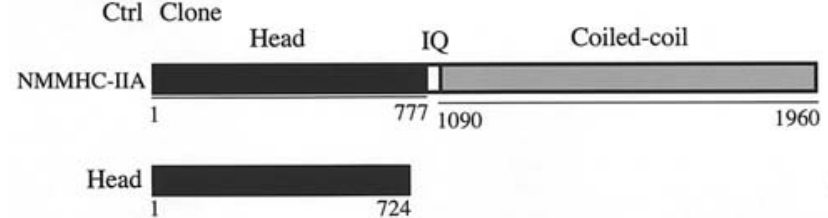

\section{-}

Coiled-coil 1

Coiled-coil 2

Figure 1. Yeast two-hybrid assay. (A) The yeast clones co-transformed with MYH9 and MYH10 cDNAs were observed as strongly activating the three reporter genes, His, Ura and LacZ, as indicated by comparison of the interaction strength with controls (CTRL) purchased by the manufacturer. (B) Direct assay of interaction between different NMMHC-A domains and the NMMHC-B coiled-coil domain. -, no interaction; ++, interaction.

rehydrated and washed in TBS, and antigen retrieval was achieved by keeping sections in sodium citrate buffer $(10 \mathrm{mM}$, $\mathrm{pH}$ 6.0) in a microwave oven at $750 \mathrm{w}$ for three 5-min passages. Slides were then pre-incubated with normal goat serum (Dako) to prevent non-specific binding and then incubated overnight at $4^{\circ} \mathrm{C}$ with CMII23 MoAb diluted 1:20 in TBS. After washing in normal saline, staining was developed with goat anti-mouse antibody Envision peroxidase (Dako) for $30 \mathrm{~min}$ (26) and diamminobenzidine (Dako) as a substrate. Before the second staining with anti-NMMHC-A, slides were pre-incubated with normal rabbit serum (Dako) to prevent non-specific binding and then incubated overnight at $4^{\circ} \mathrm{C}$ with PR-B440P antibody diluted 1:250 in TBS. After extensive washings in saline, slides were incubated for $30 \mathrm{~min}$ with swine anti-rabbit antibody (Dako) diluted 1:500 and, finally, with the strep ABC Complex/AP (Dako) diluted 1:300 for $30 \mathrm{~min}$. The reaction was revealed by BICP/NBT (Dako) as a substrate. A negative control was conducted in each experiment by replacing the specific primary antibody with non-immune serum at the same concentration.

\section{Results}

Different experimental approaches have been used to identify proteins that interact with NMMHC-A. Primary screening was carried out by the yeast two-hybrid method, followed by co-immunoprecipitation of identified interactors and immunomorphological analysis of expression in different cells.

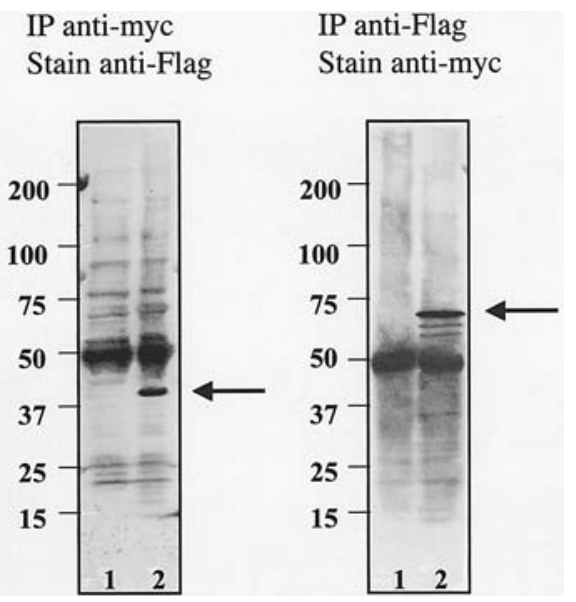

Figure 2. Co-immunoprecipitation of NMMHC-A and -B proteins in transfected cells. Proteins expressed in transfected COS-7 cells fused to epitope tags, were immunoprecipitated with anti-myc antibody, subjected to Western blot analysis and stained with anti-Flag antibody (left panel) or vice versa (right panel). The arrows indicate a $40-\mathrm{kDa}$ protein in the left panel and a $62-\mathrm{kDa}$ protein in the right panel. Migration of standard molecular weight markers is indicated in each panel. Lane 1 shows lysates from untransfected control cells, and lane 2 shows those in co-transfected cells.

Two-hybrid assay and co-immunoprecipitation. Yeast twohybrid screening was performed using the $\mathrm{C}$-terminal (fragment residues from exon 36 to 40) of NMMHC-A, including the C-terminal 1645-1960 amino acids inserted in the bait construct, and a library of HeLa cell cDNA as prey. Of $1 \times 10^{6}$ clones screened from the large-scale transformation, one was observed as strongly activating the three reporter genes, His, Ura and LacZ. The interaction strength was comparable to the yeast control purchased as the indicator of a very strong interaction (Fig. 1). Sequence analysis indicated that this clone corresponded to the C-terminal NMMHC-B, including a 1431-bp DNA fragment encoding 477 amino acids, 1168 to 1644 in the NMMHC-B tail region. To better characterize the domain of interaction, two additional constructs were used in a direct test: a more extended fragment of the NMMHC-A coiled-coil domain, including amino acids 1298 to 1960; and a fragment corresponding to amino acids 1 to 724 of the NMMHC-A head domain. Yeast cells were co-transformed with the three different $M Y H 9$ constructs and the $M Y H 1 O$ fragment corresponding to $1168-1644$ amino acids of the coiled-coil domain of NMMHC-B. No interaction took place between the NMMHC-A head domain and the NMMHC-B coiled-coil domain, while both NMMHC-A coiled-coil domain fragments were able to interact with the NMMHC-B coiled-coil domain (Fig. 1). The interaction of these two protein fragments was further analyzed by biochemical methods based on co-immunoprecipitation.

COS7 cells were co-transfected with constructs containing the C-terminal portions of MYH9 and MYH1O genes corresponding to those used in the two-hybrid method, and cloned in two different expression vectors fused to myc and Flag epitope tags, respectively. Antibodies against the epitopes were used for co-immunoprecipitation. Fig. 2 shows that proteins co-precipitated with anti-myc antibodies were stained with anti-Flag antibody and vice versa. In the former case, anti-Flag antibody revealed a protein of $40 \mathrm{kDa}$ corresponding to the cloned 1,250-bp fragment of MYH9 


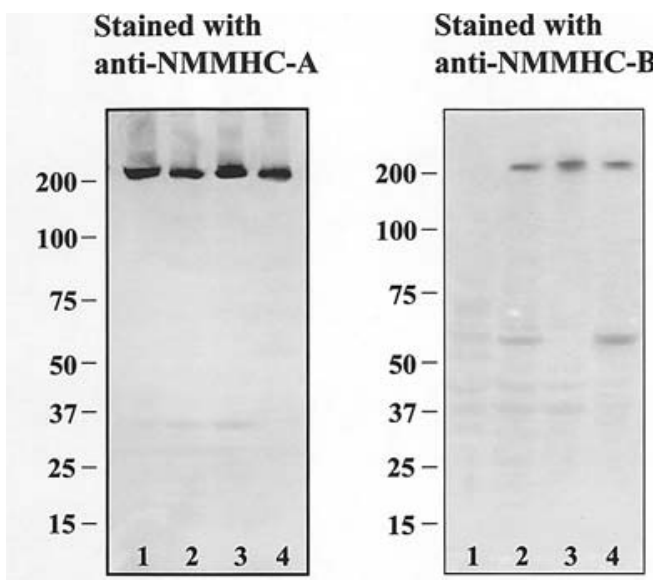

Figure 3. Expression of endogenous NMMHC-A and -B proteins in different cell types. Lysates from platelets (lane 1), HKT tubular epithelial cells (lane 2 ), cultured podocytes (lane 3 ) and skin fibroblasts (lane 4) were subjected to Western blot analysis and stained with anti-NMMHC-A, NMF6 antibody (left panel) and anti-NMMHC-B, CMII23 antibody (right panel). Molecular weight markers are indicated on the left side.

cDNA in the myc precipitate; conversely, in the anti-Flag immunoprecipitate, a protein of $62 \mathrm{kDa}$ was revealed with the anti-myc antibody corresponding to the 1,431-bp fragment of $\mathrm{MYH1O}$ cDNA. These findings confirmed the interaction between NMMHC-IIA and -IIB C-terminal tail regions found by yeast two-hybrid screening.

For co-immunoprecipitation in unmanipulated cells, we first defined the suitable cell panel. By Western blot analysis on total cell lysates, we detected expression of NMMHC-IIA in platelets, renal cells (podoctyes and tubule epithelia) and skin fibroblasts, and expression of NMMHC-IIB in the same cell types with the exception of platelets (Fig. 3). Lysates from fibroblast cells and platelets, used as negative controls not expressing $M Y H 10$, were subjected to immunoprecipitation with specific antibodies raised against NMMHC-IIA, while Western blots were stained with both anti-NMMHC-IIA and anti-NMMHC-IIB. As shown in Fig. 4, the anti-NMMHC-IIB antibody stained the immunoprecipitated protein in fibroblasts but not in platelets, thus confirming the interaction in this cell type. No signal was observed in control samples in which precipitation with protein A was performed in lysates with no antibody.

\section{Analysis of expression profiles}

Kidney and renal cells. Immumomorphological localization of NMMHC-IIA and NMMHC-IIB in the human kidney was performed on normal kidney bioptic specimens and on human kidney cell lines derived from glomerular podocytes and tubular epithelia (Figs. 5 and 6). The results of double immunostaining with antibodies against NMMHC-IIA and NMMHC-IIB of normal glomeruli and tubular epithelia are described in Fig. 5A and B, respectively. In glomeruli, both NMMHC-IIA (brown staining) and NMMHC-IIB (blue/violet staining) were detectable with areas of co-expression that appeared particularly evident in podocytes, whereas some cell types, probably corresponding to mesangial cells, appeared exclusively stained by the anti-NMMHC-IIA antibody (Fig. 5A). NMMHC-IIA and NMMHC-IIB were co-expressed in tubular epithelia (Fig. 5B).
IP anti-NMMHC-A Stain anti-NMMHC-A
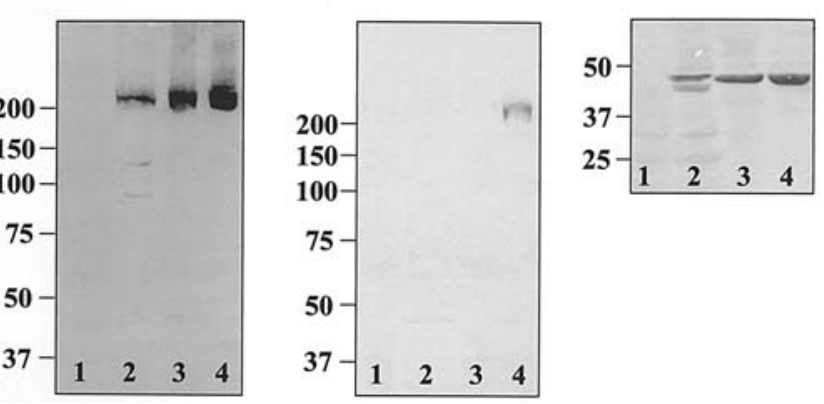

Figure 4. Co-immunoprecipitation of endogenous NMMHC-IIA and -IIB proteins. Immunoprecipitation with anti-NMMHC-IIA antibody from lysates of platelets (lanes 3) and fibroblasts (lanes 4) was analyzed by Western blotting with the same anti-NMMHC-IIA antibody (left panel), anti-NMMHCIIB antibody (central panel) and anti-actin antibody (right panel). Lane 1, protein A precipitation of a platelet lysate in the absence of antibody; lane 2, total non-immunoprecipitated lysate from platelets. Molecular weight markers are indicated on the left side.

Experiments with podocytes and tubule epithelial cells from in vitro cultures confirmed the co-expression and documented co-localization of the two isoforms. Fig. 6A and $\mathrm{B}$ shows separate staining with anti-NMMHC-A and anti-NMMHC-B antibodies of a human podocyte cell line. The NMMHC-A and -B signals appeared superimposable: both presented diffuse perinuclear staining, clearly delimitated the cell cortex and identified the same cytoplasmic filaments (indicated by arrows). Merging is shown in Fig. 6C. Analysis of fluorescence emissions on single confocal sections confirmed that the co-localization of NMMHC-IIA and -IIB signals were completely superimposable (Fig. 6D).

In HKT cells, the antibodies recognized different filaments; however, in each analyzed optical section it was possible to identify areas in which the two signals co-localized, consistent with a partial interaction of NMMHC-IIA and -IIB filaments (Fig. 6E-H). A similar pattern was also observed in cultured skin fibroblasts (Fig. 6I-L).

Bone marrow hematopoietic cells. Analysis of double-stained human bone marrow smears showed that precursors of granulocytic and megakaryocytic lineages constitutively expressed only NMMHC-IIA (not shown), while erythroid precursors synthesized both NMMHC-IIA and -IIB. In these cells, the signal of both isoforms was found to be present within limited (well-defined) zones of the cytoplasm. Confocal analysis demonstrated that the NMMHC-IIA and -IIB fluorescent signals completely co-localized in these zones in all erythroid precursor cells (Fig. 6M-P).

\section{Discussion}

Three isoforms of non-muscle myosin type II, named A, B and $\mathrm{C}$, have been identified in eukaryotic cells. Isoform $\mathrm{C}$ was discovered recently, and little information on its expression profiling and functional properties is available (27). Although isoforms $\mathrm{A}$ and $\mathrm{B}$ were identified more than 10 years ago and have been the subject of a number of studies, several aspects of these molecules are still a matter of debate. In particular, 
A

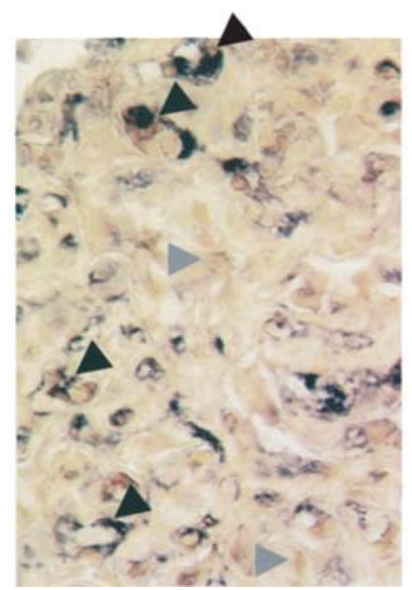

B

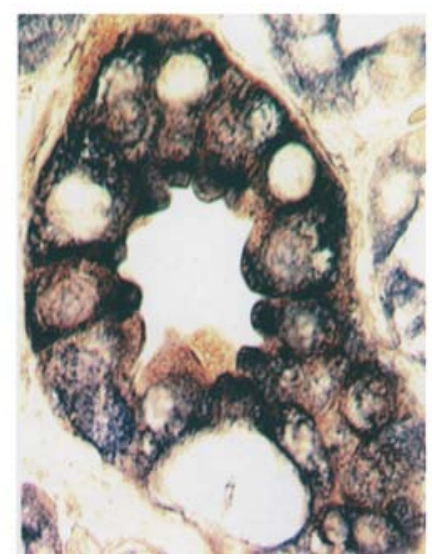

Figure 5. NMMHC-IIA and NMMHC-IIB staining in human renal biopsies. (A) Glomerular cells and (B) tubular kidney cells. Double immunostaining was obtained by utilizing specific anti-NMMHC-IIA antibody (brown staining) and anti-NMMHC-IIB antibody (blue/violet staining). Black arrows indicate cells with double staining, and grey arrows indicate cells with single brown staining.

it is not yet clear whether they play distinct roles or are functionally interconnected. Functional studies suggested that NMMHC-IIA and NMMHC-IIB present different, but complementary properties. Kinetic characterization of the two isozymes revealed that isoform A has a low duty ratio similar to that of muscle myosins, thus indicating that it spends only a small fraction of its ATPase cycle time in a strongly actinbound state (28), while isoform B is characterized by a considerably higher duty ratio. Therefore, myosin IIB is more suited for sustained maintenance of tension, and myosin IIA for short duration force generation (29). On this basis, it has been proposed that isoform $\mathrm{B}$ cooperates with isoform $\mathrm{A}$ by prolonging tension maintenance in cytoplasmic minifilamentous structures, and cofilaments of the two isoforms might exist in living cells, providing a mechanism of 'fine tuning' non-muscle myosin motor activity. The results of some immunolocalization studies are compatible with this hypothesis, since they showed that, in several cell types, myosin IIA and IIB co-localize at least in some cell areas and/or during some cell functional activities $(7,9,30)$. In other
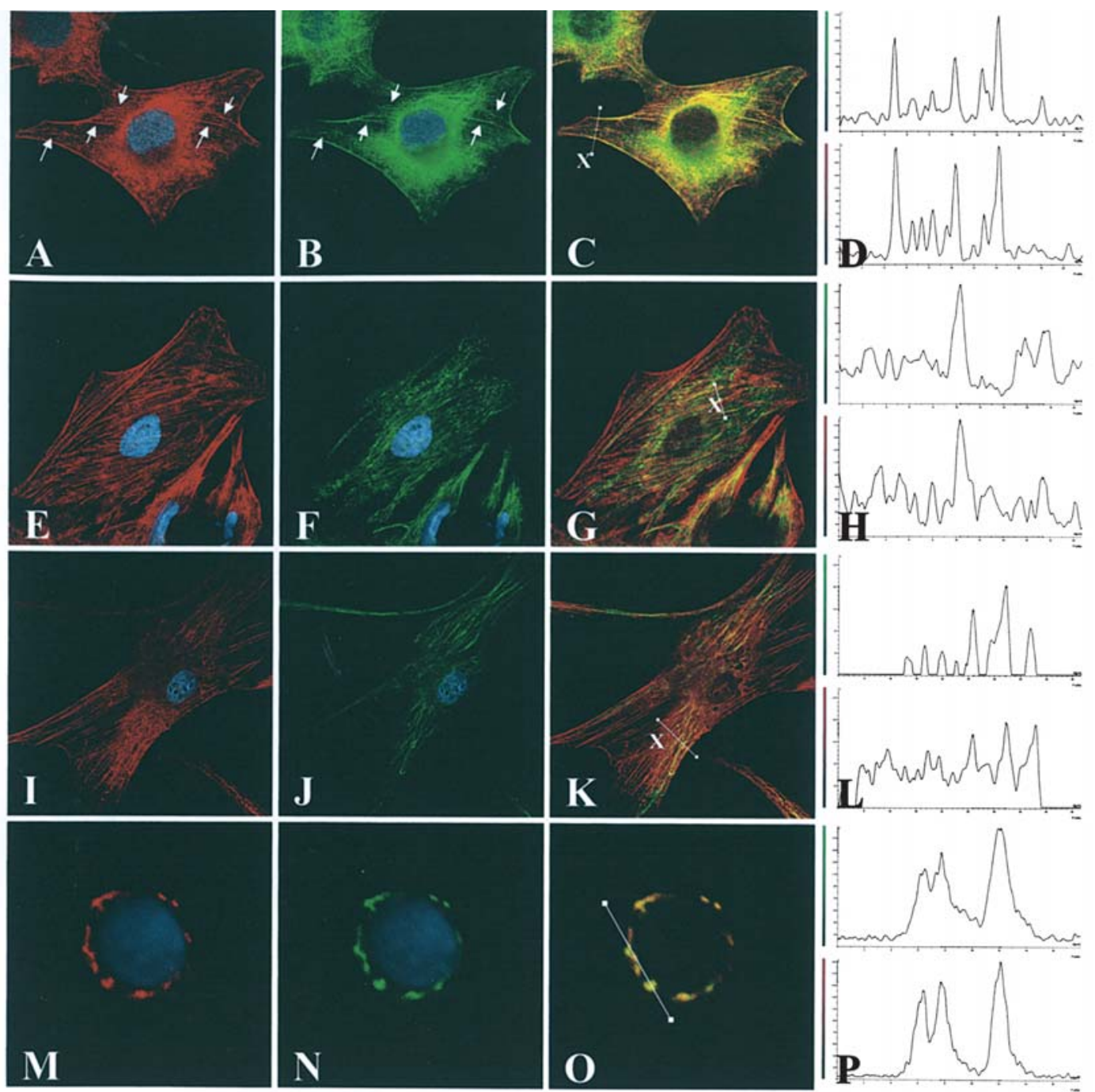

Figure 6. NMMHC-IIA and -IIB localization in different cell types. Immunofluorescence patterns of human cultured podocytes (A-D), HKT epithelial tubular cells (E-H), fibroblasts (I-L), and bone marrow erythroid precursors (M-P) are shown. Images correspond to confocal optical sections of cells double-stained for NMMHC-IIA (red) and -IIB (green). (C, G, K and O) Merging of images obtained in red and green channels. (D, H, L and P) Graphs reporting the fluorescence signal intensity along the X-rays for each fluorochrome on the optical section. 
cell models, quite different subcellular localizations of the two isoforms have been observed (8). Altogether, these results suggest that myosin isoform $\mathrm{A}$ and $\mathrm{B}$ can interact and/or cooperate in specific functions in some cell types, but direct evidence supporting this hypothesis is still lacking. Previous studies concluded that mice with a targeted deletion of the NMMHC-A encoding gene $(10,11)$ fail to develop, thus indicating an irreplaceable role of this protein during embryo development.

The present study shows that the screening of a HeLa cell cDNA library by the yeast two-hybrid system, and further characterization carried out by the same method, identified a strong interaction between the polypeptide fragment corresponding to the $\mathrm{C}$-terminal tail regions of NMMHC-B and -A. Moreover, co-immunoprecipitation experiments confirmed that the C-terminal fragments of the two isoforms bind to each other when transfected in COS-7 fibroblasts. These results for the first time demonstrate a selective and direct (i.e. not mediated by motor domains) interaction between the C-terminal rod tail domains of NMMHC-A and -B, and raise the question of the role of this interaction in living cells.

The main function of the non-muscle myosin rod tail domain is to mediate the assembly of NMMHC molecules into bipolar multimeric minifilaments, whose formation is essential for cellular functions of these molecules $(1,31)$. Thus, it seems reasonable to hypothesize that the direct interaction we found might allow the assembly of NMMHC molecules into filaments composed of both NMMHC-IIA and -IIB isoforms. In other words, NMMHC-IIA and -IIB may have retained the intrinsic capability to self-assembly during their evolution from a common ancestral molecule, despite functional specialization. In this context, co-immunoprecipitation of the constitutively expressed NMMHC-IIA and -IIB in human fibroblasts, and the complete matching of NMMHC-IIA and -IIB signals by confocal analysis in cultured renal podocytes and native bone marrow erythroblasts, support the hypothesis that heterogeneous mini-filaments could actually exist at least in some cells and in certain functional states. Of course, further studies that include immunolocalization of the two isoforms at the ultrastructural level are required to better define the characteristics of this interaction in living cells.

The finding that the NMMHC-IIB rod tail fragment is a strong molecular interactor of NMMHC-IIA is relevant, not only to cell physiology but also to the pathogenesis of MYH9-RD. As mentioned above, the same MYH9 mutations can result in quite different clinical pictures in this disorder, and it has been suggested that polymorphic variants of NMMHC-IIA interactors could make the consequences of an NMMHC-IIA defect more or less severe $(14,32)$. Since it is difficult to make rational hypotheses concerning the nature of modifier gene(s), screening methods such as the yeast twohybrid provide the initial information for further research. The disclosure that NMMHC-IIB was a strong molecular interactor of NMMHC-IIA among a large number of screened molecules indicates that it is a good candidate. A role for myosin isoforms other than NMMHC-IIA in the pathogenic mechanisms of $M Y H 9-\mathrm{RD}$ is also consistent with the expression profiles of non-muscle myosin isoforms in mouse tissue (10); isoform-IIB and/or -IIC and isoform-IIA have been identified in the kidney, lens and inner ear, which are variably affected, while only isoform-IIA was observed in megakaryocytic and granulocytic differentiation lines, which always present pathological features. On this basis, it has been hypothesized that isoform-IIB and/or -IIC compensate for the isoform A defect derived from MYH9 mutations, and delay or prevent the development of clinically relevant abnormalities. The present study demonstrates that the distribution of NMMHC-IIA and NMMHC-IIB is superimposable in human podocytes, which supports the hypothesis of functional compensation, in that ultrastructural analyses in patients with the D1424H substitution of NMMHC-IIA identified these cells as a target of NMMHC-IIA mutations in the development of kidney impairment (32). The same mechanism could also be involved in the variability of hearing and viewing defects in subjects with MYH9-RD, but the impossible task of obtaining lens and inner ear specimens from patients has so far prevented detailed studies on the cells of these organs. Finally, co-expression and co-localization of $\mathrm{A}$ and $\mathrm{B}$ isoforms in erythroid precursors and fibroblasts could explain why these cell lines do not present pathological phenotypes in MYH9-RD.

In conclusion, our investigation has provided direct evidence that NMMHC-IIA and -IIB interact in vivo by their C-terminal tail region. The close relationship between the corresponding two myosin isoforms could be functionally relevant in both physiological and pathological conditions.

\section{Acknowledgements}

We acknowledge the generous gift of immortalized podocyte cells from Professor G. Camussi, University of Torino, Italy. We thank Mrs. Loredana Velo for her secretarial assistance. This research was made possible thanks to funding from the M.I.U.R.-FIRB to R.R. and C.L.B., M.I.U.R.-PRIN to C.L.B. and M.S., and TELETHON (GP0019/01) to G.M.G., and C.L.B.

\section{References}

1. Sellers JR: Myosins: a diverse superfamily. Biochim Biophys Acta 1496: 3-22, 2000.

2. Tullio AN, Accili D, Ferrans VJ, Yu ZX, Takeda K, Grinberg A, Westpha H, Preston YA and Adelstein RS: Nonmuscle myosin II-B is required for normal development of the mouse heart. Proc Natl Acad Sci USA 94: 12407-12412, 1997.

3. Wei Q and Adelstein RS: Conditional expression of a truncated fragment of nonmuscle myosin II-A alters cell shape but not cytokinesis in HeLa cells. Mol Biol Cell 11: 3617-3627, 2000.

4. Jacobelli J, Chmura SA, Buxton DB, Davis MM and Krummel MF: A single class II myosin modulates T cell motility and stopping, but not synapse formation. Nat Immunol 5: 531-538, 2004.

5. Berg JS, Powell BC and Cheney RE: A millennial myosin census. Mol Biol Cell 12: 780-794, 2004.

6. Hodge T and Cope MJ: A myosin family tree. J Cell Sci 113: 3353-3354, 2000.

7. Maupin P, Phillips CL, Adelstein RS and Pollard TD: Differential localization of myosin-II isozymes in human cultured cells and blood cells. J Cell Sci 107: 3077-3090, 1994.

8. Kelley CA, Sellers JR, Gard DL, Bui D, Adelstein RS and Baines IC: Xenopus nonmuscle myosin heavy chain isoforms have different subcellular localizations and enzymatic activities. J Cell Biol 134: 675-687, 1999.

9. Kolega J: Cytoplasmic dynamics of myosin IIA and IIB: spatial 'sorting' of isoforms in locomoting cells. J Cell Sci 111: 2085-2095, 1998. 
10. Conti MA, Even-Ram S, Liu C, Yamada KM and Adelstein RS: Defects in cell adhesion and the visceral endoderm following ablation of nonmuscle myosin heavy chain II-A in mice. J Biol Chem 279: 41263-41266, 2004.

11. Matsushita T, Hayashi H, Kunishima S, Hayashi M, Ikejiri M, Takeshita K, Yuzawa Y, Adachi T, Hirashima K, Sone M, Yamamoto K, Takagi A, Katsumi A, Kawai K, Nezu T, Takahashi M, Nakashima T, Naoe T, Kojima T and Saito H: Targeted disruption of mouse ortholog of the human MYH9 responsible for macrothrombocytopenia with different organ involvement: hematological, nephrological, and otological studies of heterozygous $\mathrm{KO}$ mice. Biochem Biophys Res Commun 24: 1163-1171, 2004.

12. Lalwani AK, Goldstein JA, Kelley MJ, Luxford W, Castelein CM and Mhatre AN: Human nonsyndromic hereditary deafness DFNA17 is due to a mutation in nonmuscle myosin MYH9. Am J Hum Genet 67: 1121-1128, 2000.

13. Donaudy F, Snoeckx R, Pfister M, Zenner HP, Blin N, Di Stazio M, Ferrara A, Lanzara C, Ficarella R, Declau F, Pusch CM, Nurnberg P, Melchionda S, Zelante L, Ballana E, Estivill X, Van Camp G, Gasparini P and Savoia A: Nonmuscle myosin heavy-chain gene MYH14 is expressed in cochlea and mutated in patients affected by autosomal dominant hearing impairment (DFNA4). Am J Hum Genet 74: 770-776, 2004.

14. Seri M, Pecci A, Di Bari F, Cusano R, Savino M, Panza E, Nigro A, Noris P, Gangarossa S, Rocca B, Gresele P, Bizzaro N, Malatesta P, Koivisto PA, Longo I, Musso R, Pecoraro C, Iolascon A, Magrini U, Rodriguez Soriano J, Renieri A, Ghiggeri GM, Ravazzolo R, Balduini CL and Savoia A: MYH9related disease: May-Hegglin anomaly, Sebastian syndrome, Fechtner syndrome, and Epstein syndrome are not distinct entities but represent a variable expression of a single illness. Medicine 82: 203-215, 2003.

15. Kawamoto S and Adelstein RS: Chicken nonmuscle myosin heavy chains: differential expression of two mRNAs and evidence for two different polypeptides. J Cell Biol 112: 915-924, 1991.

16. Marigo V, Nigro A, Pecci A, Montanaro D, Di Stazio M, Balduini CL and Savoia A: Correlation between the clinical phenotype of MYH9-related disease and tissue distribution of class II nonmuscle myosin heavy chains. Genomics 83: 1125-1133, 2004.

17. Phillips CL, Yamakawa K and Adelstein RS: Cloning of the cDNA encoding human nonmuscle myosin heavy chain -B and analysis of human tissues with isoform-specific antibodies. J Muscle Res Cell Motil 16: 379-389, 1995.

18. Takeda K, Kishi H, Ma X, Yu ZX and Adelstein RS: Ablation and mutation of nonmuscle myosin heavy chain II-B results in a defect in cardiac myocyte cytokinesis. Circ Res 93: 330-337, 2003.

19. Conrad AH, Jaffredo T and Conrad GW: Differential localization of cytoplasmic myosin II isoforms A and B in avian interphase and dividing embryonic and immortalized cardiomyocytes and other cell types in vitro. Cell Motil Cytoskel 31: 93-112, 1995
20. Candiano G, Gusmano R, Altieri P, Bertelli R, Ginevri F, Coviello DA, Sessa A, Caridi G and Ghiggeri GM: Extracellu1ar matrix formation by epithelial cells from human polycystic kidney cysts in culture: Virchow Arch B Cell Pathol Incl Mol Pathol 63: 1-9, 1992.

21. Conaldi PG, Biancone L, Bottelli A, De Martino A, Camussi G and Toniolo A: Distinct pathogenic effects of group B coxsackie viruses on human glomerular and tubular kidney cells. J Virol 71: 9180-9187, 1997.

22. Conaldi PG, Biancone L, Bottelli A, Wade-Evans A, Racusen LC, Boccellino M, Orlandi V, Serra C, Camussi G and Toniolo A: HIV-1 kills renal tubular epithelial cells in vitro by triggering an apoptotic pathway involving caspase activation and Fas upregulation. J Clin Invest 102: 2041-2049, 1998

23. Laemmli UK: Cleavage of structural proteins during the assembly of the head of bacteriophage T4. Nature 227: 680-685, 1970

24. Candiano G, Musante L, Bruschi M, Ghiggeri GM, Herbert B, Antonucci $F$ and Righetti PG: Two-dimensional maps in soft immobilized $\mathrm{pH}$ gradient gels: a new approach to the proteome of the Third Millennium. Electrophoresis 23: 292-297, 2002.

25. Van der Loos CM, Naruko T and Becker AE: The use of enhanced polymer one-step staining reagents for immunoenzyme double-labelling. Histochem J 28: 709-714, 1996.

26. Sabattini E, Bisgaard K, Ascani S, Poggi S, Piccioli M, Ceccarelli C, Pieri F, Fraternali-Orcioni G, and Pileri SA: The EnVision $^{++}$system: a new immunohistochemical method for diagnostics and research. Critical comparison with the APAAP, ChemMate, CSA, LABC, and SABC techniques. J Clin Pathol 51: 506-511, 1998.

27. Golomb E, Ma X Jana SS, Preston YA, Kawamoto S, Shoham NG, Goldin E, Conti MA, Sellers JR and Adelstein RS: Identification and characterization of nonmuscle myosin II-C, a new member of the myosin II family. J Biol Chem 279: 2800-2808, 2004.

28. Kovacs M, Wang F, Hu A, Zhang Y and Sellers JR: Functional divergence of human cytoplasmic myosin II: kinetic characterization of the non-muscle IIA isoform. J Biol Chem 278: 38132-38140, 2003 .

29. Wang Q, Moncman CL and Winkelmann DA: Mutations in the motor domain modulate myosin activity and myofibril organization. J Cell Sci 116: 4227-4238, 2003.

30. Saitoh T, Takemura S, Ueda K, Hosoya H, Nagayama M, Haga H, Kawabata K, Yamagishi A and Takahashi M: Differential localization of non-muscle myosin II isoforms and phosphorylated regulatory light chains in human MRC fibroblasts. FEBS Lett 509: 365-369, 2001

31. Ikebe M, Komatsu S, Woodhead J, Mabuchi K, Ikebe R, Saito J, Craig R and Higashihara M: The tip of the coiled-coil rod determines the filament formation of smooth muscle and nonmuscle myosin. J Biol Chem 276: 30293-30300, 2001.

32. Ghiggeri GM, Caridi G, Magrini U, Sessa A, Savoia A, Seri M, Pecci A, Romagnoli R, Gangarossa S, Noris P, Sartore S, Necchi V, Ravazzolo R and Balduini CL: Genetics, clinical and pathological features of glomerulonephritis associated with mutations of nonmuscle myosin IIA (Fechtner syndrome). Am J Kidney Dis 41: 95-110, 2003. 